PROCEEDINGS OF THE

AMERICAN MATHEMATICAL SOCIETY

Volume 125, Number 12, December 1997, Pages 3593-3599

S 0002-9939(97)03997-X

\title{
POINT SPECTRUM AND MIXED SPECTRAL TYPES FOR RANK ONE PERTURBATIONS
}

\author{
RAFAEL DEL RIO AND BARRY SIMON
}

(Communicated by Palle E. T. Jorgensen)

\begin{abstract}
We consider examples $A_{\lambda}=A+\lambda(\varphi, \cdot) \varphi$ of rank one perturbations with $\varphi$ a cyclic vector for $A$. We prove that for any bounded measurable set $B \subset I$, an interval, there exist $A, \varphi$ so that $\left\{E \in I \mid\right.$ some $A_{\lambda}$ has $E$ as an eigenvalue agrees with $B$ up to sets of Lebesgue measure zero. We also show that there exist examples where $A_{\lambda}$ has a.c. spectrum $[0,1]$ for all $\lambda$, and for sets of $\lambda$ 's of positive Lebesgue measure, $A_{\lambda}$ also has point spectrum in $[0,1]$, and for a set of $\lambda$ 's of positive Lebesgue measure, $A_{\lambda}$ also has singular continuous spectrum in $[0,1]$.
\end{abstract}

\section{$\S 1$. INTRODUCTION}

In this note we will consider families of operators

$$
A_{\lambda}=A+\lambda(\varphi, \cdot) \varphi
$$

where $A$ is a self-adjoint operator on a separable Hilbert space $\mathcal{H}$ and $\varphi \in \mathcal{H}$ is a cyclic vector for $A$. It will be convenient to consider also the value $\lambda=\infty$, which is the operator $Q A Q$ on $Q \mathcal{H}$ where $Q$ is the projection onto the operators orthogonal to $\varphi$. Let $d \mu_{\lambda}$ be the spectral measure for $A_{\lambda}$ with vector $\varphi$ and $d \rho_{\lambda}=\left(1+\lambda^{2}\right) d \mu_{\lambda}$. It it known [3] that $d \rho_{\lambda}$ has a weak limit as $\lambda \rightarrow \infty, d \rho_{\infty}$, which is a spectral measure for $A_{\infty}$.

Define for $x \in \mathbb{R}$,

$$
G_{\lambda}(x)=\int \frac{d \rho_{\lambda}(y)}{(x-y)^{2}}
$$

where $G$ may be infinite.

Also define for $z \in \mathbb{C}$ with $\operatorname{Im} z>0$,

$$
F_{\lambda}(z)=\int \frac{d \rho_{\lambda}(E)}{E-z}=(1+\lambda)^{2}\left(\varphi,\left(A_{\lambda}-z\right)^{-1} \varphi\right) .
$$

(This differs from the standard $F[6]$ by a factor of $\left(1+\lambda^{2}\right)$.) It is known [2], [6] that

Received by the editors July 3, 1996.

1991 Mathematics Subject Classification. Primary 47A55.

This material is based upon work supported by the National Science Foundation under Grant No. DMS-9401491. The Government has certain rights in this material.

The first author was partially supported by CONACYT, Project 400316-5-0567PE. 
Theorem 0. The sets

$$
\begin{aligned}
& P=\left\{E \mid G_{\lambda}(E)<\infty\right\} \cup\left\{E \mid E \text { is an eigenvalue of } A_{\lambda}\right\}, \\
& L=\left\{E \mid \lim _{\epsilon \downarrow 0} F_{\lambda}(E+i \epsilon) \equiv F_{\lambda}(E+i 0) \text { exists and } \operatorname{Im} F_{\lambda}(E+i 0)>0\right\}, \\
& S=\mathbb{R} \backslash P \cup L
\end{aligned}
$$

are $\lambda$ independent for $\lambda \in \mathbb{R}$, and for every $\lambda \in \mathbb{R} \cup\{\infty\}$ :

$$
\begin{aligned}
& \rho_{\lambda}^{\mathrm{pp}}(\cdot)=\rho_{\lambda}(\cdot \cap P), \\
& \rho_{\lambda}^{\mathrm{ac}}(\cdot)=\rho_{\lambda}(\cdot \cap L), \\
& \rho_{\lambda}^{\mathrm{sc}}(\cdot)=\rho_{\lambda}(\cdot \cap S),
\end{aligned}
$$

where $\rho_{\lambda}^{\mathrm{pp}}, \rho_{\lambda}^{\mathrm{ac}}, \rho_{\lambda}^{\mathrm{sc}}$ are the pure point, absolutely continuous, and singular continuous parts of the measure $\rho_{\lambda}$. Moreover,

$$
P=\bigcup_{\lambda \in \mathbb{R} \cup\{\infty\}}\left\{E \mid E \text { is an eigenvalue of } A_{\lambda}\right\}
$$

and for any set $C$,

$$
\int \frac{\rho_{\lambda}(C)}{\left(1+\lambda^{2}\right)} d \lambda=|C|
$$

the Lebesgue measure of $C$. In particular, by (1a)

$$
\int \frac{\rho_{\lambda}^{\mathrm{pp}}(C)}{\left(1+\lambda^{2}\right)} d \lambda=|C \cap P|
$$

and similarly for $L$ and $S$.

One can ask what kind of sets can occur as a $P$. We have a partial answer given in Section 2:

Theorem 1. For any bounded measurable set $B$ and any interval $I \supset B$, there exists a measure $d \mu$ on I so that (where a.e. means with respect to Lebesgue measure)

$$
G_{0}(x)= \begin{cases}<\infty & \text { a.e. } x \in B, \\ =\infty & \text { a.e. } x \in I \backslash B .\end{cases}
$$

The measure d $\mu$ may be chosen purely a.c., or purely s.c., or purely p.p.

Remarks. 1. By Theorem 0, this says something about allowed sets of eigenvalues.

2. We will also show that if $B$ is open, we can drop the a.e. We believe that this can be done for an arbitrary $F_{\delta}$, but have not proven it.

Using a technical result in Section 3, we will prove our second main result in Section 4:

Theorem 2. There exists an example $A$ so that

(i) $\sigma_{\mathrm{ac}}\left(A_{\lambda}\right)=[0,1]$ for all $\lambda$.

(ii) $\left\{\lambda \mid \sigma_{\mathrm{pp}}\left(A_{\lambda}\right) \cap[0,1] \neq \emptyset\right\}$ has positive Lebesgue measure; indeed, for any interval $I \subset[0,1],\left\{\lambda \mid \sigma_{\mathrm{pp}}\left(A_{\lambda}\right) \cap I \neq \emptyset\right\}$ has positive measure.

(iii) $\left\{\lambda \mid \sigma_{\mathrm{sc}}\left(A_{\lambda}\right) \neq \emptyset\right\}$ has positive Lebesgue measure; indeed, for any interval $I \subset[0,1],\left\{\lambda \mid \sigma_{\mathrm{sc}}\left(A_{\lambda}\right) \cap I \neq \emptyset\right\}$ has positive measure.

There also exist examples where (i) is replaced by $\sigma_{\mathrm{ac}}\left(A_{\lambda}\right)=\emptyset$. 
One can translate these results into ones for variations on boundary conditions for Schrödinger operators $-u^{\prime \prime}+V u$ on $[0, \infty)$ in two steps:

(a) Extend the theory to $\varphi \in \mathcal{H}_{-1}(A)$ and rewrite the Sturm-Liouville/Schrödinger operator in this language [6].

(b) Appeal to the Gel'fand-Levitan construction [5], which implies that for any measure $\mu$ on a bounded interval $I$, we can find a continuous $V$ on $[0, \infty)$ with $-u^{\prime \prime}+V u$ limit point at infinity and boundary condition $\theta$ at $x=0$ so that the spectral measure $d \rho_{\theta}$ restricted to $I$ is $d \mu$. Typical of the result is:

Theorem 1'. For any bounded measurable set $B$ and interval $I \supset B$, there is a continuous function $V$ on $[0, \infty)$ so that up to sets of Lebesgue measure zero,

$$
\left\{E \mid-u^{\prime \prime}+V u=E u \text { has a solution } L^{2} \text { at infinity }\right\}
$$

is precisely $B$.

Because the Gel'fand-Levitan construction gives no information on $V$ at infinity (for example, it could be unbounded below), we regard these translations as being of limited interest.

\section{$\S 2$. The SET Where $\boldsymbol{G}$ IS FINITE}

Recall that a perfect set is a closed set with no isolated points. We will also need the following notion.

Definition. A closed subset $C \subset \mathbb{R}$ will be called minimal if and only if for all $x \in C$ and $\epsilon>0,|(x-\epsilon, x+\epsilon) \cap C|>0$.

The name comes from the fact that among all closed sets $D$ with $|D \triangle C|=0, C$ is the minimal such set. We will see below that any closed set $D$ has a minimal closed set $C$ contained in it so that $|D \backslash C|=0$.

We also define $G_{\mu}$ by

$$
G_{\mu}(x)=\int \frac{d \mu(y)}{(x-y)^{2}} .
$$

With these notions out of the way, we can state the two main theorems of this section:

Theorem 2.1. (a) Let $C$ be any closed set in $\mathbb{R}$. Then there exists a pure point measure $\mu$ supported on $C$ so that $\left\{x \mid G_{\mu}(x)=\infty\right\}=C$.

(b) Let $C$ be any perfect set. Then there exists a singular continuous measure $\mu$ supported on $C$ so that $\left\{x \mid G_{\mu}(x)=\infty\right\}=C$.

(c) Let $C$ be any minimal closed set. Then there exists an absolutely continuous measure $\mu$ supported on $C$ so that $\left\{x \mid G_{\mu}(x)=\infty\right\}=C$.

Remarks. 1. The assumptions on the closed sets are optimal in that if $x$ is an isolated point of $C$, then $G_{\mu}(x)<\infty$ for any singular continuous measure $\mu$ supported on $C$; and if $x \in C$ is a point with $|(x-\epsilon, x+\epsilon) \cap C|=0$ for some $\epsilon>0$, then $G_{\mu}(x)<\infty$ for any a.c. measure supported on $C$.

2. In general, $\left\{x \mid G_{\mu}(x)=\infty\right\}$ is only a $G_{\delta}$, not a closed set. It is open if "closed" in this theorem can be replaced by $G_{\delta}$.

3. If $B$ is any measurable set, we can apply the methods of proof below and get a $\mu$ supported on $B$ with $\left\{x \mid G_{\mu}(x)=\infty\right\} \supset B$. If $B$ is arbitrary, we can take $\mu$ pure point. If $B$ has no isolated points, we can take $\mu$ singular continuous, and if 
$B$ has no essentially isolated points (i.e., no points $x$ with $|(x-\epsilon, x+\epsilon) \cap B|=0$ for some $\epsilon>0$ ), we can take $\mu$ absolutely continuous.

If we are willing to throw out sets of measure zero, we can go beyond Theorem 2.1. We write $A \equiv B$ to mean $|A \triangle B|=0$. Then we will prove that:

Theorem 2.2 (三 Theorem 1). For $B$ an arbitrary measurable subset of an interval $I$, we can find $\mu$ supported on $I$ so that

$$
\left\{x \in I \mid G_{\mu}(x)<\infty\right\} \equiv B .
$$

$\mu$ can be chosen to be purely absolutely continuous or purely singular continuous or pure point. In the a.c. case, $\mu$ can be chosen so that the essential support of $\mu$ is $I \backslash B$.

In understanding perfect and minimal closed sets, it is useful to have the following pair of results, which we will also need in proving Theorem 2.2.

Proposition 2.3. Any closed set $S$ in $\mathbb{R}$ can be written as $S=C \cup D$ where $C$ is perfect and $D$ is countable.

Proof. Let $C=\{x \in S \mid \forall \epsilon>0,(x-\epsilon, x+\epsilon) \cap S$ is uncountable $\}$ and $D=S \backslash C$. It is easy to see that $C$ is closed. If we show $D$ is countable, then each $(x-\epsilon, x+\epsilon) \cap C$ is uncountable, so not empty and $C$ is perfect.

If $x \notin C$, we can find $a$ and $b$ rational so $x \in(a, b)$ and $(a, b) \cap S$ is countable. Since there are only countably many $(a, b)$ with $a, b$ rational, we can find a countable family of $\left\{O_{n}\right\}_{n=1}$ with each $O_{n} \cap S$ countable, so $D \subset \cup_{n}\left(O_{n} \cap S\right)$ is countable.

Proposition 2.4. Any closed set $S$ in $\mathbb{R}$ can be written as $S=C \cup D$ where $C$ is minimal closed and $|D|=0$.

Proof. Let $C=\{x \in S|\forall \epsilon>0|,(x-\epsilon, x+\epsilon) \cap S \mid>0\}$ and $D=S \backslash C$. Now just mimic the proof of Proposition 2.3.

We need one more preliminary:

Proposition 2.5. (a) For any non-empty closed set $C$, there exists a point measure supported by $C$.

(b) For any non-empty perfect set $C$, there exists a singular continuous measure supported by $C$.

(c) For any non-empty minimal closed set $C$, there is an absolutely continuous measure supported by $C$.

Proof. (a) is trivial and stated for parallelism. (c) is also trivial (take $d \mu=\chi_{C} d x$ ). That leaves (b); so let $C$ be perfect. If $C$ contains an entire interval $[a, b]$, place a scaled Cantor measure on $(a, b)$ and use that for $d \mu$. So we need only consider a nowhere dense perfect set. By intersecting it with a suitable bounded interval and scaling, we will suppose it is a subset of $[0,1]$.

We claim such a $C$ is homeomorphic to $\{0,1\}^{\mathbb{N}}$, the infinite sequences of 0 's and 1 's. Use that homeomorphism to transfer the two mutually singular measures

$$
d \alpha_{1}=\bigotimes_{n=1}^{\infty}\left[\frac{1}{2}\left(\delta_{0}+\delta_{1}\right)\right] \text { and } \quad d \alpha_{2}=\otimes_{n=1}^{\infty}\left(\frac{1}{3} \delta_{0}+\frac{2}{3} \delta_{1}\right)
$$


$d \alpha_{1}$ may be purely absolutely continuous (as it is if $C$ is a symmetric positive measure Cantor set), but then $d \alpha_{2}$ is purely singular continuous. Either way, either $d \alpha_{1}$ or $d \alpha_{2}$ has a non-zero singular continuous component.

To prove the claim (known, but the proof is so short that we give it) that a nowhere closed perfect subset $C$ of $[0,1]$ is homeomorphic to $\{0,1\}^{\mathbb{N}}$, let $a_{-}=$ $\min (C), a_{+}=\max (C)$, and $\ell_{1}=a_{+}-a_{-}$, the length of $C$. Since $C$ is perfect, $\ell_{1}>0$. Let $J=\left(\frac{a_{-}+a_{+}}{2}-\frac{\ell_{1}}{6}, \frac{a_{-}+a_{+}}{2}+\frac{\ell_{1}}{6}\right)$, the middle third of $\left(a_{-}, a_{+}\right)$. Since $C$ is nowhere dense, we can find $x_{1} \in J \backslash C$. Let $C_{0}=C \cap\left(-\infty, x_{1}\right), C_{1}=C \cap\left(x_{1}, \infty\right)$. Then $C_{0}, C_{1}$ are perfect and $\operatorname{diam}\left(C_{1}\right) \leq \frac{2}{3}$. Now repeat this process, and so find $C_{m_{1} \ldots m_{\ell}}\left(m_{i} \in\{0,1\}\right)$ inductively so that $\operatorname{diam}\left(C_{m_{1} \ldots m_{\ell}}\right) \leq\left(\frac{2}{3}\right)^{\ell}, C_{m_{1} \ldots m_{\ell}}=$ $C_{m_{1} \ldots m_{\ell} 0} \cup C_{m_{1} \ldots m_{\ell} 1}$, each $C_{m_{1} \ldots m_{\ell}}$ is perfect. Define $a_{\ell}: C \rightarrow\{0,1\}$ by $a_{\ell}=0$ on each $C_{m_{1} \ldots m_{\ell-1} 0}$ and $a_{\ell}=1$ on each $C_{m_{1} \ldots m_{\ell-1} 1}$. Each $a_{\ell}$ is continuous since each $C_{m_{1} \ldots m_{\ell}}$ is closed. Map $C \rightarrow\{0,1\}^{\ell}$ by $x \rightarrow\left(a_{1}(x), a_{2}(x), \ldots\right)$. This map is onto since for any fixed $m_{1}, \ldots, \bigcap_{\ell=1}^{\infty} C_{m_{1} \ldots m_{\ell}} \neq \emptyset$ by compactness. This map is one-one since $\operatorname{diam}\left(C_{m_{1} \ldots m_{\ell}}\right) \rightarrow 0$ to $\ell \rightarrow \infty$ uniformly in the choice of $m_{\ell}$. A continuous bijection is a homeomorphism.

Proof of Theorem 2.1. This is motivated by a construction in [7]. For $n=1,2, \ldots$ and $j=0, \ldots, 2^{n}-1$, let $C_{j}^{(n)}=\overline{\left(\frac{j}{2^{n}}, \frac{j+1}{2^{n}}\right) \cap C}$ which is $C \cap\left[\frac{j}{2^{n}}, \frac{j+1}{2^{n}}\right]$ with the endpoints dropped if they would be isolated. Then if $C$ is perfect (minimal), so is each non-empty $C_{j}^{(n)}$. For such non-empty $C_{j}^{(n)}$, let $\mu_{j}^{(n)}$ be a measure of the requisite type (i.e., pure point, singular continuous, or absolutely continuous) of unit measure and supported on $C_{j}^{(n)}$. Such measures exist by Proposition 2.5. Let

$$
\mu=\sum_{n=1}^{\infty} n^{-2} 2^{-n} \sum_{\substack{j=1 \\ j \text { so that } \\ C_{j}^{(n)} \neq \emptyset}}^{2^{n}} \mu_{j}^{(n)} .
$$

Then $\mu$ is a finite measure of the requisite type supported on $C$. If $y \notin C$, then $G_{\mu}(y) \leq \operatorname{dist}(y, C)^{-2} \int d \mu<\infty$ since $C$ is closed. On the other hand, if $y \in C$ and $y \in\left(\frac{j}{2^{n}}, \frac{j+1}{2^{n}}\right)$, then $C_{j}^{(n)} \neq \emptyset$ and $\int \frac{d \mu_{j}^{(n)}(x)}{(x-y)^{2}} \geq\left(2^{-n}\right)^{2}$, and if $y \in\left\{\frac{j}{2^{n}}\right\}_{j=0}^{2^{n}} \cap C$, either $C_{j}^{(n)}$ or $C_{j-1}^{(n)}$ is non-empty. It follows that

$$
\int \frac{d \mu(x)}{(x-y)^{2}} \geq \sum_{n=1}^{\infty} 2^{2 n} n^{-2} 2^{-n}=\infty
$$

so $\left\{y \mid G_{\mu}(y)=\infty\right\}=C$.

Proof of Theorem 2.2. This uses an explicit version of an argument of Howland [4] as in [1]. Since Lebesgue measure is inner regular, we can find $C_{1}, \ldots C_{n}, \ldots$ and $K_{1}, \ldots, K_{n}, \ldots$ closed with $C_{1} \subset C_{2} \subset \cdots \subset I \backslash B$ and $K_{1} \subset K_{2} \subset \cdots \subset B$ and with $\left|B \backslash \cup_{n} K_{n}\right|=0,\left|(I \backslash B) \backslash \cup_{n} C_{n}\right|=0$.

By Proposition 2.3, we can suppose that $C_{n}$ 's are minimal closed (and so, perfect) without loss of generality. We can also suppose each $C_{n}$ is non-empty (if $|I \backslash B|=0$, wejust take $\mu=0)$. 
Let $\mu_{n}$ be a unit measure of the requisite type supported on $C_{n}$ with

$$
C_{n}=\left\{x \mid G_{\mu_{n}}(x)=\infty\right\}
$$

Let

$$
\mu=\sum_{n=1}^{\infty} 2^{-n} \operatorname{dist}\left(K_{n}, C_{n}\right)^{2} \mu_{n} .
$$

Since $K_{n}$ and $C_{n}$ are compact and disjoint, $\operatorname{dist}\left(K_{n}, C_{n}\right)>0$ and thus, $G_{\mu}(x) \geq$ $2^{-n} \operatorname{dist}\left(K_{n}, C_{n}\right)^{2} G_{\mu_{n}}(x)=\infty$ on $C_{n}$ and so on $\cup C_{n}$ and so a.e. on $I \backslash B$.

On the other hand, since $K_{n} \subset K_{n+1}, \ldots, \operatorname{dist}\left(K_{n}, C_{m}\right) \geq \operatorname{dist}\left(K_{m}, C_{m}\right)$ if $m \geq n$ and so if $x \in K_{n}$,

$$
G_{\mu}(x)=\sum_{\ell=1}^{n-1} 2^{-\ell} \operatorname{dist}\left(K_{\ell}, C_{\ell}\right)^{2} G_{\mu_{\ell}}(x)+\sum_{\ell=n}^{\infty} 2^{-n}<\infty,
$$

and so $G_{\mu}<\infty$ on $\cup K_{n}$ and thus a.e. on $B$.

In the a.c. case, we can take $\mu_{n}=\frac{1}{\left|C_{n}\right|} \chi_{C_{n}} d x$, in which case it is evident that the essential support of $\mu$ is $\cup C_{n}=I \backslash B$ as claimed.

\section{§3. Essentially Dense SETS}

Definition. A measurable set $S \subset I$, an interval, is called essentially dense if for every subinterval $J \subset I$, we have $|J \cap S|>0$.

Theorem 3.1. There exist disjoint measurable subsets $A, B, C \subset[0,1]$ whose union is $[0,1]$ so that each is essentially dense.

Remarks. 1. Our proof shows that one can assert the same for sets $A_{1}, \ldots, A_{n}$ rather than three sets or even construct a countable disjoint decomposition, each of which is essentially dense.

2. Our construction is related to a construction in [2].

Proof. Let $n_{j}=(2 j+1)^{2}$, the square of the $j^{\text {th }}$ odd number. Given $x \in[0,1]$, we define $a_{j}(x)$ by requiring

$$
x=\sum_{j=1}^{\infty} \frac{a_{j}(x)}{n_{1} \ldots n_{j}}
$$

with $a_{j}(x) \in\left\{0,1, \ldots, n_{j}-1\right\}$ and the requirement that if $x$ 's expansion can end in all 0 's, we do that (to settle the ambiguity between $\ldots a\left(n_{j}-1\right)\left(n_{j+1}-1\right) \ldots$ and $\ldots(a+1) 00 \ldots)$. This is a standard positive measure Cantor set construction. Define $m_{j}=\frac{1}{2}\left(n_{j}-1\right)$. Let

$$
\begin{aligned}
& A=\left\{x \mid \text { the number of } j \text { 's with } a_{j}(x)=m_{j} \text { is } 1,4, \ldots \text { or infinite }\right\}, \\
& B=\left\{x \mid \text { the number of } j \text { 's with } a_{j}(x)=m_{j} \text { is } 2,5,8, \ldots\right\}, \\
& C=\left\{x \mid \text { the number of } j \text { 's with } a_{j}(x)=m_{j} \text { is } 3,6,9, \ldots\right\} .
\end{aligned}
$$

This is obviously a decomposition. We need only to show that each set is essentially dense. It suffices to show that $|B \cap J|>0$ for any interval of the form $J=\{x \mid$ $\left.a_{1}(x)=\alpha_{1}, \ldots, a_{k}(x)=\alpha_{k}\right\}$ since every interval contains such a $J$. By increasing $k$ by 1 or 2 and shrinking $J$ by taking $\alpha_{k+1}=m_{k+1}$ (and perhaps $\alpha_{k+2}=m_{k+2}$ ), we can suppose that $\#\left\{j \in\{1, \ldots, k\} \mid \alpha_{j}=m_{j}\right\} \equiv 2 \bmod 3$. In that case, by 
looking at $x$ 's with no further $a_{\ell}(x)=m_{\ell}$, we have

$$
|B \cap J| \geq \prod_{\ell=k+1}^{\infty}\left(1-\frac{1}{n_{\ell}}\right)>0
$$

since $\sum \frac{1}{n_{\ell}}<\infty$.

\section{$\S 4$. MiXED SPECTRA}

Proof of Theorem 2. Decompose $[0,1]=A \cup B \cup C$ into three disjoint essentially dense sets. Pick a measure $d \mu_{1}$ which is absolutely continuous with essential support $A$ so that $G_{\mu_{1}}(x)<\infty$ a.e. on $B \cup C$ and a s.c. measure $\mu_{2}$ supported on $B$ so that $G_{\mu_{2}}(x)<\infty$ on $A \cup C$ and $G_{\mu_{2}}(x)=\infty$ a.e. on $B$. Let $d \mu=d \mu_{1}+d \mu_{2}$.

By Theorem 0 (recall $X \equiv Y$ means $|X \triangle Y|=0$ ),

$$
\begin{aligned}
P & \equiv C \cup(\mathbb{R} \backslash[0,1]), \\
L & \equiv A, \\
S & \equiv B .
\end{aligned}
$$

By equation (3) and its analogs for a.c. and s.c., we have the claimed assertions (i)-(iii). For the examples with $\sigma_{\mathrm{ac}}\left(A_{\lambda}\right)=\emptyset$, just use $d \mu=d \mu_{2}$.

\section{ACKNOWLEDGMENT}

R.d.R. would like to thank Professor Alejandro Bravo for very useful discussions.

\section{REFERENCES}

1. R. del Rio, S. Jitomirskaya, Y. Last, and B. Simon, Operators with singular continuous spectrum, IV. Hausdorff dimensions, rank one perturbations, and localization, J. d'Analyse Math. 69 (1996), 153-200. CMP 97:06

2. R. del Rio, B. Simon, and G. Stolz, Stability of spectral types for Sturm-Liouville operators, Math. Research Lett. 1 (1994), 4R37-450 MR 95i:47084

3. F. Gesztesy and B. Simon, Rank one perturbations at infinite coupling, J. Funct. Anal. 128 (1995), R245-252 MR 95m:47014

4. J. Howland, On a theorem of Carey and Pincus, J. Math. Anal. 145 (1990), 562-565 MR 91f:47020; CMP 97:01

5. B.M. Levitan, Inverse Sturm-Liouville Problems, VNU Science Press, Utrecht, 1987. MR 89b:34001

6. B. Simon, Spectral analysis of rank one perturbations and applications, in CRM Proc. and Lecture Notes, (J. Feldman, R. Froese, and L. Rosen, eds.), Vol. 8, pp. 109-149, Amer. Math. Society, Providence, RI, 1995. MR 97c:47008

7. B. Simon and T. Wolff, Singular continuous spectrum under rank one perturbations and localization for random Hamiltonians, Commun. Pure Appl. Math. 39 (1986), 911-918 MR 87k:47032

Iimas-Unam, Apdo. Postal 20-726, Admon. No. 20, Deleg Alvaro Obregon, 01000 Mexico, MeXico

Division of Physics, Mathematics, and Astronomy, California Institute of Technology, Pasadena, California 91125 